\title{
Stably stratified layers within Earth's core
}

\section{Journal Article}

Author(s):

Hardy, Colin (1); Wong, Jenny

Publication date:

2019-06

Permanent link:

https://doi.org/10.3929/ethz-b-000520577

Rights / license:

In Copyright - Non-Commercial Use Permitted

Originally published in:

Astronomy \& Geophysics 60(3), https://doi.org/10.1093/astrogeo/atz148 


\section{Stably stratified layers within Earth's core}

\section{Colin M Hardy and Jenny}

Wong review whether the Earth's core contains stratified layers, which has huge implications for the geodynamo.

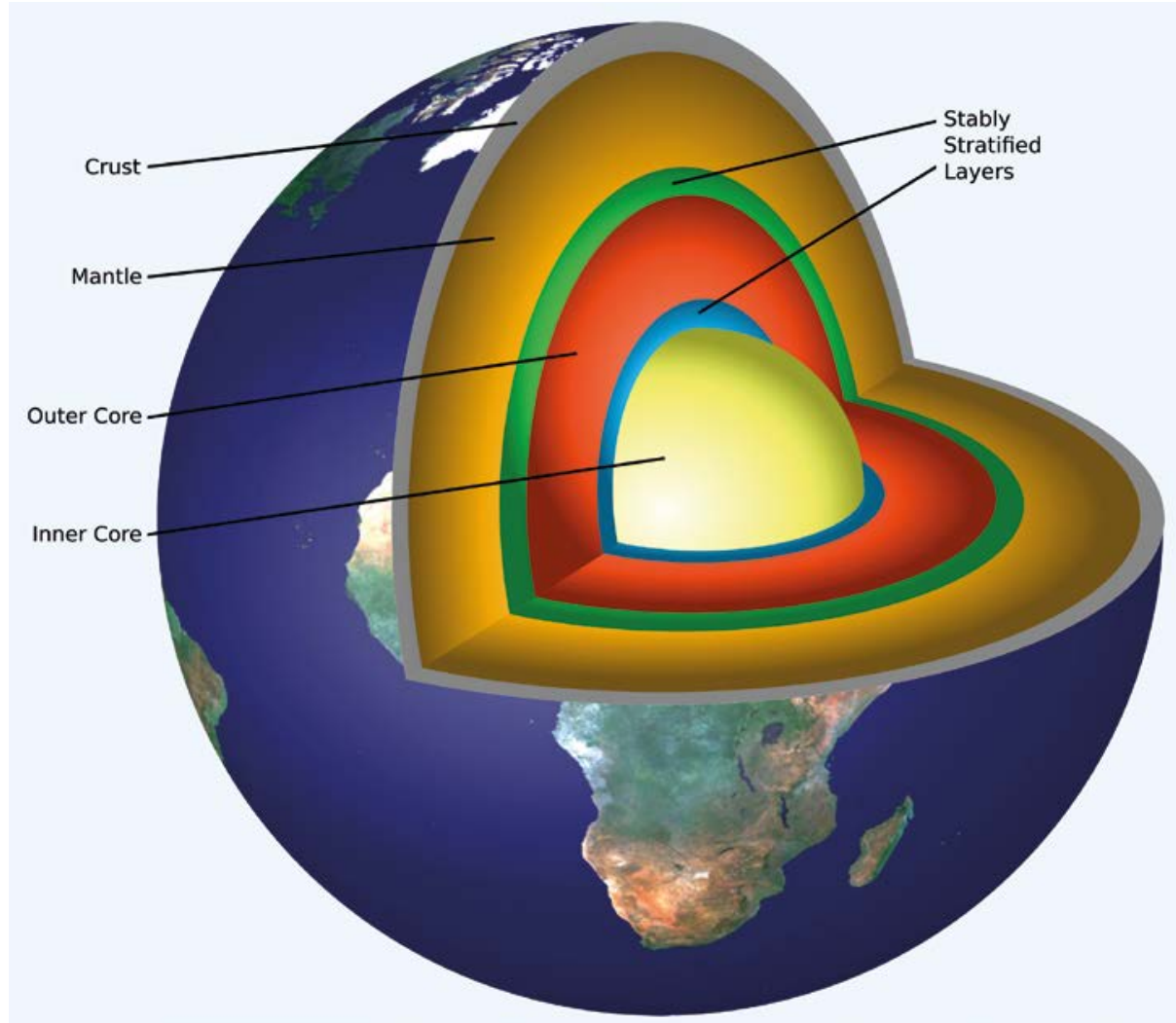

1 Earth's interior, showing the location of the proposed stratified layers within the Earth's core.

planet like an invisible force field, protecting life from harmful solar radiation by deflecting charged particles that would otherwise damage satellites and cause major disruption to our communication services. A proper understanding of how the geomagnetic field is generated relies on a detailed knowledge of the behaviour within the core region. This enigmatic dynamo mechanism, which is extremely sensitive to core conditions, is a very active research topic and one of the greatest outstanding problems in Earth science.

Planetary dynamos work by the same principles as the standard school physics experiment in which you move a coil of wire around a permanent magnet. Faraday's law of induction describes how an electromotive force pushes electrons through the metal, creating an electric current in the wire. Within Earth's outer core, convection of electrically conducting molten iron generates electric currents in the presence of a magnetic field. The rotation of the Earth breaks spherical symmetry, so that these currents favour motion parallel to the rotation axis. Hence magnetic fields are produced that combine to create one large magnetic field engulfing our planet. One of the main sources of power driving this geodynamo is the slow cooling of the Earth, as heat left over from its formation is lost at the surface. This flow of internal heat drives vigorous convection in the metallic liquid outer core, thus powering the dynamo that sustains the magnetic field. Thermal and compositional convection are assumed to take place throughout the liquid outer core, except in thin boundary layers at the top and bottom; more detail on the convection process within the core is covered in the review by Jones (2015). The Earth's core is mainly composed of iron (around $85 \%$ by weight), and the rest - a significant fraction of its mass - consists of a poorly constrained combination of lighter elements including silicon, nickel, sulphur, oxygen, carbon and hydrogen (Nimmo 2007). It is conventionally assumed that vigorous convection leaves the outer core in a well-mixed, because of the role the magnetic field plays in our current way of life. It surrounds our 
2 Image of the radial magnetic field at the coremantle boundary based on the observational satellite data for 2015 from the Chaos 6 model by Finlay et al. (2016)

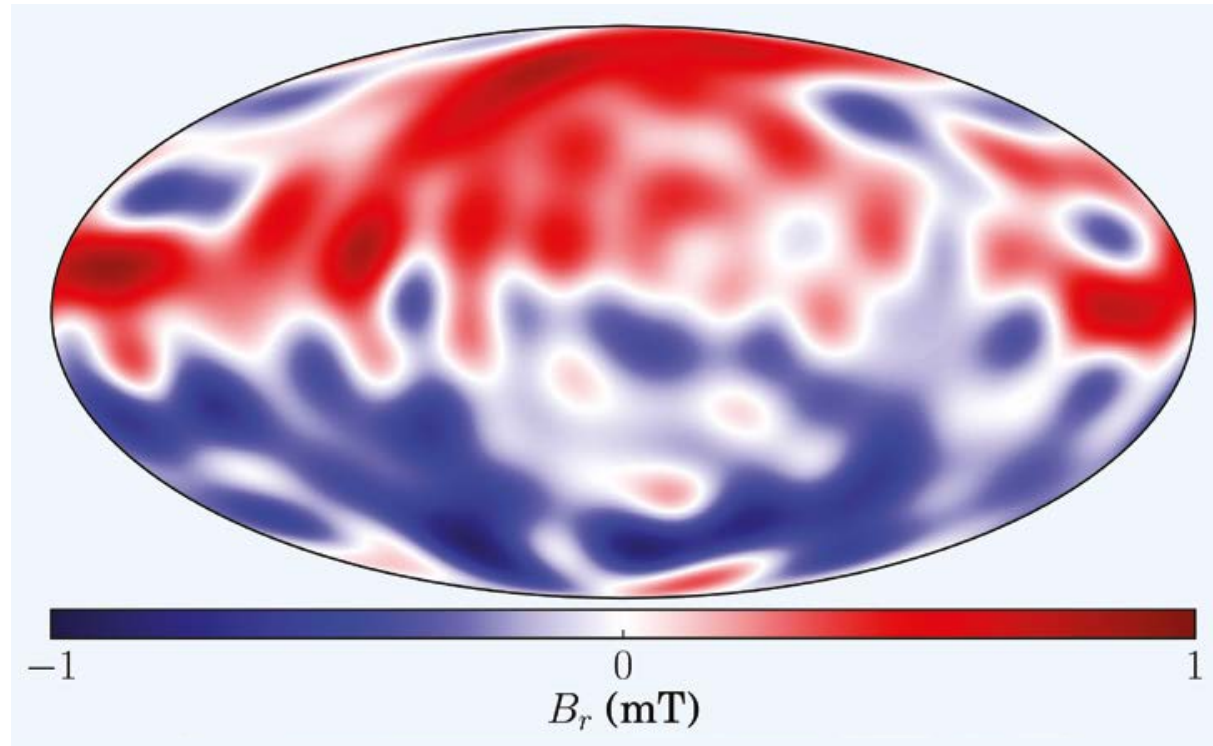

distributed over the surface of the Earth. High-resolution data are also available from satellites such as SWARM, ESA's three spacecraft that orbit the planet together recording the field. Conductivity in the mantle is very small, which means that it can be considered to be electrically insulating, allowing the surfacial field to be continued downwards to obtain an accurate and observationally derived picture of the magnetic field at the core-mantle the bottom and lighter at the top. Stably stratified fluids are ubiquitous in Nature, and within geophysics the most comprehensively studied applications are within atmospheric and oceanic fluid dynamics (Vallis 2017). While it is relatively simple to observe Earth's stratified atmosphere, which divides naturally into layers based on characteristics such as temperature and composition, it is more challenging to identify such layers within Earth's core. This is largely because thousands of kilometres of rock lie between us and the core, inhibiting our ability to make direct measurements of the temperature, pressure and composition.

\section{Core dynamics}

If stratified layers do exist within Earth's core, then they could have profound effects on core dynamics and our understanding of many geophysical phenomena. Stably stratified fluids are dynamically very different from convecting regions. A key distinctive feature inherent to stratified layers is the suppression of radial motion of the fluid. Radial motion is crucial for the radial heat transport within the core, that powers the geodynamo. This motion is important in the bulk of the liquid core, therefore any layers within which no radial fluid motion exists would have profound consequences for the nature of Earth's dynamo.

Observations of Earth's magnetic field are made at many ground stations dynamo and core evolution. It would raise important questions about how light elements that are released during inner core growth can contribute to compositional convection on the other side of this layer.

Dynamics at these two extremes locations - the very top and bottom of the inner core - can fundamentally impact on each other. Mantle temperatures at the CMB are not uniform because of the complex nature of mantle convection (Tackley 2007); this means that the heat fluxes out of the core may have significant lateral variations. This inhomogenous heat flux distribution is sufficiently strong that simulations (Gubbins et al. 2011) show its propagation throughout the core, leading to non-uniform temperatures at the inner core boundary (ICB). This can result in simultaneous freezing and melting at different places on the ICB, which has been proposed as a mechanism for the formation of a stratified layer at the base of the inner core. This demonstrates one example of how these two stratified layers, often treated as separate entities, may be intrinsically linked. Another comes from the motion of light elements within the core. Light elements released at the ICB as solid iron freezes are buoyant and rise through the liquid core. Understanding how light elements can traverse through the lower stratified layer without disturbing it is vital for comprehending core convection. This can have a direct effect on the top of the core because it may be expected that some of the light elements will eventually rise all the way up to the $\mathrm{CMB}$ where they can collect (Moffatt \& Loper 1994), in the same way that cigarette smoke collects at the top of a room. This forms a compositionally stratified layer underneath the $\mathrm{CMB}$, consisting of iron with a higher proportion of light elements.

\section{Discovering the Earth's interior}

In 1889, at Potsdam, Von Reuber Pachwitz used a low-frequency instrument (intended 
to collect tide data), to record an earthquake that took place in Japan (Souriau 2007).

This first recording of a remote earthquake opened up the possibility of sampling the structure of the deep Earth using teleseismic data (measurement of a tremor caused by an earthquake far away). Seismic waves are waves of energy that travel through the Earth. Teleseismic records show two distinct first arrivals: a P wave and then an $S$ wave. The propagation velocity of the waves depends on the density and elasticity of the medium they are travelling through. An understanding of these waves and their measurement allows properties of the Earth's internal structure to be deduced. During the 20th century, the field of seismology made many notable discoveries that have increased our knowledge of the complex, non-uniform nature of Earth's structure. Oldham (1906) identified the existence of a distinct core through measurements of the delayed arrival times of $S$ waves and, within a few years, Gutenberg (1913) had built on this work to determine the depth of the CMB to an extraordinarily accurate estimate of $2900 \mathrm{~km}$, a value extremely close the current best estimate of $2891 \mathrm{~km}$ (Dziewonski \& Anderson 1981). Lehmann

\section{"Seismic evidence soon mounted up, claiming layer thicknesses up to 350 km"}

describing the Earth's average properties, delineates the ICB as a sharp interface with no stratified layers.

Interest in the $\mathrm{F}$ layer reignited after seismic investigations by Souriau and Poupinet (1991a), who used PKPbc waves to directly sample the lowermost outer core (figure 3). They discovered an anomalous layer roughly $200 \mathrm{~km}$ thick, with a slower P wave velocity than expected from PREM. Through the relation

$$
v_{\mathrm{p}}^{2}=K / \rho
$$

where $v_{\mathrm{p}}$ is the $\mathrm{P}$ wave speed, $K$ is the bulk modulus and $\rho$ is the density, Souriau and Poupinet (1991a) inferred the presence of a stably stratified layer increasing in density with depth, assuming that the bulk modulus was more or less constant at pressures near that of the ICB. Seismic evidence soon mounted up, claiming layer thicknesses up to $350 \mathrm{~km}$ (Zou et al. 2008), and a new F layer was incorporated into later standard reference models such as PREM2 (Song \& Richards 2006) and ak135 (Kennett et al. 1995) that typically reduced the $P$ wave velocity in the F layer by $0.8 \%$ compared with PREM.

The density jump across the ICB is another important indicator of the existence of the F layer. There are two types of data used to core itself is not continuous, through the detection of $\mathrm{P}$ waves in a region called the "shadow zone" where no P waves would arrive without reflection at a discontinuity within the core. Her work showed that there exists an inner core with different properties compared to the rest of the core. In more recent years, both the accuracy and resolution of seismic data has increased, such that the layered core-mantle system is seen to exhibit a non-uniform structure within each region (Kind \& Li 2015, Souriau 2007). These sublayers can have important effects on the whole system, in particular within the core where the dynamics are crucial to dynamo action.

\section{The base of the core}

Early seismological models distinguished what was called an F layer with a sharp discontinuity alongside the base of the outer core. Bullen's original naming convention placed this stratified layer between the $\mathrm{E}$ and $G$ layers of the outer core and inner core respectively (Bullen 1953, Jeffreys 1939). Later work showed that the F layer identified then was actually an artifact of waves scattered by the $\mathrm{D}^{\prime \prime}$ layer in the lowermost portion of the mantle (Cleary \& Haddon 1972, King et al. 1973); the F layer then disappeared from seismic pictures of the Earth's interior. For instance, the Preliminary Reference Earth Model (PREM), a widely used one-dimensional model constrain this: normal modes, which are long-period, free oscillations of the Earth (think of the Earth as a ringing bell), and short-period body waves, which provide greater resolution than normal modes, but in principle are less exact than normal modes (Deuss 2014). Long-period waves are sensitive to the average properties between the inner and outer core, whereas shortperiod waves sample the density jump at the ICB itself. Ab initio studies suggest that the density change when pure iron melts is $240 \mathrm{~kg} \mathrm{~m}^{-3}$ (Alfè et al. 2002b); by comparing the normal mode and body wave estimates, this suggests that the density difference cannot be explained by phase change alone and the remainder of the density contrast must be compositional in origin. Masters and Gubbins (2003) used normal mode data to show that the density jump at the ICB is $\Delta \rho_{\text {mod }}=820 \pm 180 \mathrm{~kg} \mathrm{~m}^{-3}$. Studies using body wave data report that the density jump can be as little as $\Delta \rho_{\text {mod }}=520 \pm 240 \mathrm{~kg} \mathrm{~m}^{-3}$ (Koper \& Pyle 2004), and as high as $\Delta \rho_{\text {mod }}=1100 \mathrm{~kg} \mathrm{~m}^{-3}$ (Tkalčić et al. 2009). some of these seismic estimates and how they pertain to the degree of density stratication in the $\mathrm{F}$ layer.

\section{The top of the core}

Whether there is a stratified layer at the outermost part of core has long been debated (Bergman 1993, Braginsky 1984, There still remains great uncertainty over

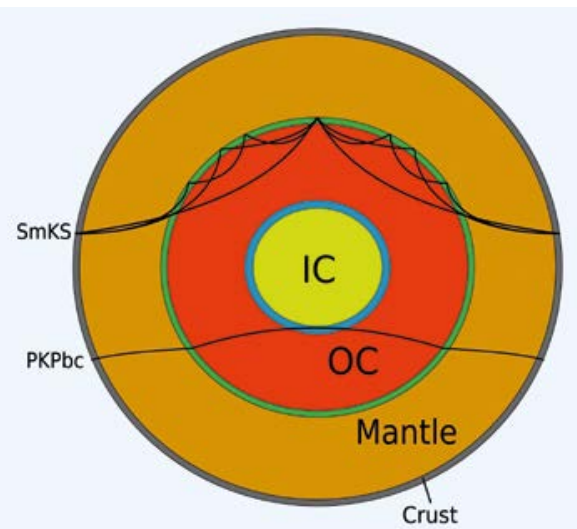

3 Schematic of Earth's interior, which illustrates the paths through the core taken by both SmKS and PKPbc waves.

1987). Seismic body wave measurements of smaller wave velocities in this region immediately beneath the $\mathrm{CMB}$, rather than across the bulk of the core, have led to proposals that the outermost part of the core has a different density and/or elasticity than the rest of the core. But this evidence is far from conclusive, with contrasting results from different seismic studies.

A large number of studies observed this deviation in the travel times of SmKS waves that pass through this layer several times as they undergo multiple reflections at the $\mathrm{CMB}$, as shown in figure 3 . Most estimates of the thickness of the stratified layer lie between approximately $200 \mathrm{~km}$ (Helffrich \& Kaneshima 2013, Lay \& Young 1990) and $300 \mathrm{~km}$ (Helffrich \& Kaneshima 2010), but some are as large as $800 \mathrm{~km}$ (Kaneshima 2018, Souriau \& Poupinet 1991b).

While a perturbation in velocity is expected within a layer of differing composition, the sign of the observed change is problematic. The velocity measured is lower than the rest of the core, which is the opposite to what we might expect if the density is smaller, because wave velocity is inversely proportional to the square root of density (equation 1). This is not well understood.

It is possible that the elastic properties of liquid mixtures can behave non-intuitively because they do not mix in ideal ways. Helffrich (2012) finds that under certain conditions, wave speeds in liquids decrease as more of a "faster" component is added. Theoretically this means that the assumption of a linear variation of wave speed with concentration is generally invalid, but the high-temperature, high-pressure conditions in Earth's core would be expected to inhibit any non-ideal mixing. Brodholt and Badro (2017) estimate the composition required to explain seismic observations at the top of the core. When considering the conditions present at the $\mathrm{CMB}$, they find that increasing the concentration of any light elements always increases velocity and so a low-velocity and low-density 
layer cannot be made by simply increasing light element concentration. While a lowvelocity and low-density layer could exist at the top of Earth's core, it would require a delicate combination of an increase in the concentration of some light elements and a relative decrease in others.

The difficulty in explaining the conclusions drawn from seismological observations around the $\mathrm{CMB}$ may lead to questions about their validity. There are inherent difficulties in inverting seismic wave travel times to determine wave velocities within this remote but relatively shallow region. In fact some argue that the velocities estimated agree with the wellmixed model of PREM to within observational uncertainties. Alexandrakis and Eaton (2010) claim that there is insufficient evidence for an anomalous layer at the top of the core. Also, the latest estimates of the elastic parameters of the outer core from Irving et al. (2018) predict a steeper wave velocity gradient with depth in the core, which could explain the SmKS wave travel times without requiring an anomalously slow layer at the top of the core. Hence, while the majority of current seismic evidence is in favour of this stratified layer, there is no unequivocal evidence.

\section{Stably stratified layer at the top of the core}

Various mechanisms have been suggested to explain why the upper reaches of the core could have become stably stratified, either compositionally or thermally. A buoyant compositional layer could form by the accumulation of light elements beneath the CMB. Light material could be transferred inwards across the $\mathrm{CMB}$ or created by chemical reaction with the mantle. Alternatively, light elements could rise within the core by the process of barodiffusion (the tendency of light elements to diffuse along a pressure gradient) after being either expelled outwards from the inner core, or precipitated out of the bulk of the outer core mixture (Gubbins \& Davies 2013). But barodiffusion alone cannot be solely responsible because this would result in a high-velocity, low-density layer, rather than the low-velocity, low-density layer observed. Additionally, because we know so little about the early Earth, we cannot rule out the possibility of inheritance. Perhaps the stratified layer is a remnant of the early Earth, resulting from incomplete mixing in the core following a major impact. This possibility was recently explored in depth by Landeau et al. (2016), who also suggest that the F layer at the base of the inner core may be another vestige of core formation. A compositionally stratified layer would be stable to convection from fluid below (Buffett \& Seagle 2010) and would grow at the rate determined by the ratio of the net supply of light elements and any entrainment from the bulk.

At the top of the core, thermal heterogeneity can arise even without chemical or boundary effects. If current estimates of the thermal conductivity of iron (which are significantly larger than previously thought) are correct (Pozzo et al. 2012), then the adiabatic heat flux at the CMB is higher than most estimates based on mantle convection models. This creates sub-adiabatic conditions at the top of the core, meaning a thermally stratified layer must exist. The complex interaction of both thermal and chemical effects may be important in the formation of this layer. For more details on

......... this see the recent thermochemical model by Nakagawa (2018), or the review by Davies et al. (2015).

A stratified layer at the top of the liquid core supports distinct waves that in principle are observable. One such class are magnetostrophic (MAC) waves, which arise from the interplay between magnetic, buoyancy (Archimedean) and Coriolis forces in equilibrium. These waves typically have periods on timescales of $10^{4}-10^{5}$ years, in the absence of a stratified layer. However, stratified layers are dynamically very different from convecting regions; they suppress radial motion and support a different suite of waves, which means that if a stably stratified layer exists at the top of the outer core it would introduce MAC waves of much shorter timescales (Braginsky 1993). Buffett (2014) modelled these waves and his work appears to confirm Braginsky's idea by finding MAC waves with a period of 60 years. This is consistent with observations of secular variation in the geomagnetic field that have identified periodic fluctuations on a period of 60 years (Roberts et al. 2007). This adds to the weight of evidence that the top of the fluid core is stably stratified and, in turn, that this affects the magnetic field.

But how much does a stratified layer affect the magnetic field signal? The stratified layer near the CMB would act as a spatial filter of the magnetic field signal, because it would place the convective region further from the surface and reduce the non-axisymmetric field generation in the dynamo region (Christensen 2006, Christensen \& Wicht 2008). Hence stably stratified layers at the top of the core enhance the dipole component of the surface magnetic field relative to smaller-scale components. This means that, although the dominantly dipolar nature of Earth's field is intrinsic to the dynamo mechanism, a stratified layer would magnify its preeminence in observations.
This is important for modelling core dynamics because measurements of the Earth's magnetic field are continued downward to the $\mathrm{CMB}$, where it is interpreted as the magnetic field at the outer edge of the convective region that is generating the dynamo action. But the presence of a stratified layer below the CMB means this field may differ from the field in the convective region; this raises the question of whether any CMB heterogeneity - topographical, electromagnetic or thermal - would penetrate the stratified layer or be damped?

Such a layer may disconnect the Ekman boundary layer from the free-stream flow, leading to a partial or even full suppression of the boundary-driven zonal flow, as a result of a competition between stratification and boundary forcing that results from heterogeneous outer boundary conditions (Cox et al. 2018).

Additionally, it has been suggested that on top of the passive attenuation effect, stable layers can also actively produce small-scale surface fields. Numerical dynamo simulations that include a stratified layer at the top of the core demonstrate the impact that stratification strength has on the morphology of the resultant magnetic field (Olson et al. 2018). The inclusion of a thin stable layer in dynamo models can destabilize the dynamo by generating a thermal wind that changes the differential rotation pattern within the core (Stanley \& Mohammadi 2008).

\section{Geomagnetic observations}

Despite the evidence for the presence of this stratified layer outlined above, there is still no consensus on its existence largely because of a contradiction with magnetic field measurements. Geomagnetic observations find reverse flux patches, strong local variations in magnetic field strength where the magnetic field in a small region has opposite polarity to the dominant polarity in that hemisphere. These regions grow or shrink and move with time. This secular variation can be explained only by radial flows immediately beneath the $\mathrm{CMB}$, which are accelerated locally or by very strong poloidal field gradients (Gubbins 2007). However, radial flows are not expected to exist in stratified layers, so it is difficult to explain geomagnetic observations if a stratified layer does exist at the top of the core. Gubbins (2007) shows that the toroidal flux expulsion associated with the observed secular variation of flux patches requires vertical flow within $100 \mathrm{~km}$ of the core surface in the absence of steep poloidal field gradients immediately beneath the CMB - for which no convincing justification has been made. This suggests an upper limit on the thickness of this layer, at least in the regions where reverse flux patches exist. 
Lesur et al. (2015) examined whether it is possible to produce the observed secular variation from a large-scale purely toroidal flow, permissible within a stratified layer. They found that in this idealized case of pure toroidal flow and no diffusion it is not possible to fit the CHAMP satellite data; it is possible that including diffusion may result in an acceptable fit, but diffusion has timescales much longer than the observed field evolution, making a fit that holds over long timescales challenging. However, including a small amount of poloidal flow can produce models that accurately describe secular variation observations. Although stratification may be strong, it would not entirely prevent all poloidal flow, so it is not possible to conclude that secular variation observations violate the hypothesis that the outermost part of the core is stably stratified.

Reconciling geomagnetic observations with seismological evidence remains extremely challenging. One possible hypothesis is that there is a non-global stratified layer, which instead of spanning the whole CMB, leaves space for regions of active radial flows (Mound et al. 2017). More research is required.

\section{The F layer}

The thicknesses of layers observed by seismology are much greater than thermal diffusion length scales, suggesting that the F layer cannot be a conductive boundary layer: other non-diffusive processes must be involved. Reconciling the F layer with traditional models of the core heat flow is problematic. Geochemical arguments suggest that silicon, sulphur and oxygen are likely candidates for the light elements in the core. Alfè et al. (2002a) showed that silicon and sulphur both partition evenly into the solid inner core and liquid outer core during solidification, but oxygen goes almost entirely into the liquid, readily providing the buoyancy needed to drive compositional convection. Additionally, constraints on the mass of the core and the density jump across the ICB must be satisfied by core composition. Hirose et al. (2013) agree that oxygen is an essential ingredient of the core, and first principles calculations by Badro et al. (2014) support this. Recently, hydrogen has been ruled out as the single light element in the core because inner core shear velocities cannot be reconciled with its presence (Umemoto \& Hirose 2015), and the amount of carbon in the core deduced from sound velocity measurements is too small to make up for the core density deficit (Nakajima et al. 2015).

But if the F layer exists, how can light elements pass into the overlying, convecting core while preserving the stable layer? How did the layer develop to begin with and how is it sustained? Answering these questions
4 Cartoon of the inner-core convective translation model.

5 Cartoon of the slurry model.

6 Cartoon of the mush model.

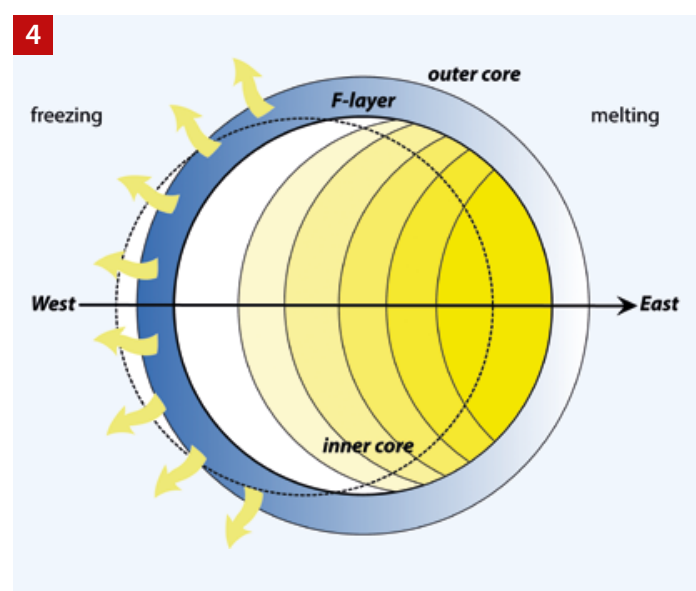

may considerably affect current understanding of how the geodynamo derives its compositional power - a major power source.

\section{Models of the F layer}

Current research directed around explaining the geodynamics of the $F$ layer is multipronged, with three main themes: convective translation, slurries and mushes (figures 4, 5 and 6). Each model has their own merits and pitfalls, a characteristic trait of an active research area.

Inner core translation (figure 4) arises from convectively unstable conditions inside the inner core, where asymmetry is caused by freezing on the western hemisphere and melting on the eastern hemisphere. In their models, Alboussière et

"The question of how
the F layer came into
existence remains
open"

dynamical model of the original F layer based on a slurry mechanism, using an iron sulphur alloy on the sulphur-rich side of the eutectic. Loper and Roberts (1977) developed an extensive general slurry theory derived from fundamental thermodynamic principles, though this was not directly applied to the F layer we know of today. Following the evidence for the $\mathrm{F}$ layer emerging from later seismic studies, Gubbins et al. (2008) attempted to explain the structure using a thermochemical model that considered thermal and compositional effects. This study led to solutions with the correct stratification, but a complete dynamical explanation of how the light element is transported through a stable layer remained elusive. Wong et al. al. (2010) use both a low thermal conductivity that favours superadiabatic conditions for thermal convection, and a high critical viscosity on the order of $10^{18}$ Pas to reduce deformation and promote convective instability. This mechanism may be limited by more recent high thermal conductivity estimates (de Koker et al. 2012, Gomi et al. 2013, Pozzo et al. 2012) that suggest that more heat is conducted out of the inner core and less energy is available to drive convection. This implies that the inner core is thermally stratified rather than convecting. In response, Deguen et al. (2018) consider double diffusive convection in the inner core because of the large difference in thermal and chemical diffusivities. They propose that inner core translation is feasible provided that the compositional profile of the inner core is destabilizing and that the inner core viscosity is sufficiently large, which they conclude was likely over the history of the inner core but unlikely to be true in the present day.

Proponents of the slurry F layer model (figure 5) suppose that crystals of solid iron form some distance above the ICB then move inwards under gravity, settling like snow to form and grow the inner core. Braginsky (1963) developed the first
(2018) introduce the effect of crystallizing small amounts of a solid phase so that light material is released above the ICB, where solid iron forms and snows onto the inner core. This was achieved by simplifying the general non-equilibrium theory of Loper and Roberts (1977) and steady-state solutions to the simplified slurry model were calculated, in which select parameters produced stably stratified layers.

The slurry model (figure 6) assumes that the inner core grows by solid particles settling at the ICB under gravity. Alternatively, the inner core may grow through a mushy layer, where supercooling above the ICB promotes the interfacial crystal growth of solid iron (Fearn et al. 1981). The resulting mushy solid matrix is permeated by liquid channels that are enriched in the residual light element, sometimes called chimneys (Mullins \& Sekerka 1964). The mushy assemblage of solid possesses a higher fraction of solid compared with the grainy iron snow particles of the slurry model. Deguen et al. (2007) study the linear stability of a mush to find that the interdendritic spacing is several metres at the ICB, with an approximate mush thickness of $300 \mathrm{~km}$ that extends down into the inner core. It is unclear whether seismic evidence 


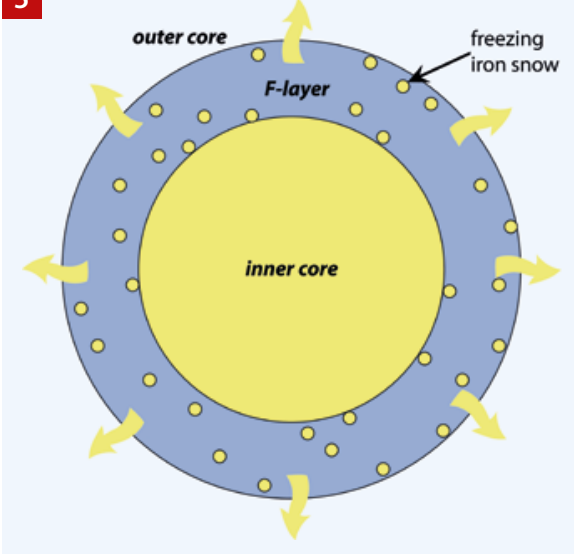

is consistent with a mostly solid, mushy F layer several hundreds of kilometres thick.

Though the slurry, mush and convective translation models may explain a stably stratified layer, the question of how the $F$ layer came into existence remains open. Currently there is interest in how the first stable iron crystal nucleated to form the inner core; the problem is that the activation energy barrier for homogeneous nucleation requires implausibly high supercooling on the order of $1000 \mathrm{~K}$, which is paradoxically so large that the inner core can never freeze out (Davies et al. in press, Huguet et al. 2018). Is it possible that stable stratification is a primordial feature of the core (Landeau et al. 2016)? Is the layer currently in a state of growth or will the layer recede over time? Will the layer remain stable in the future? The $F$ layer remains an enigmatic feature of the core and its effect on overall core dynamics and energy transfer in the geodynamo remains to be investigated.
6

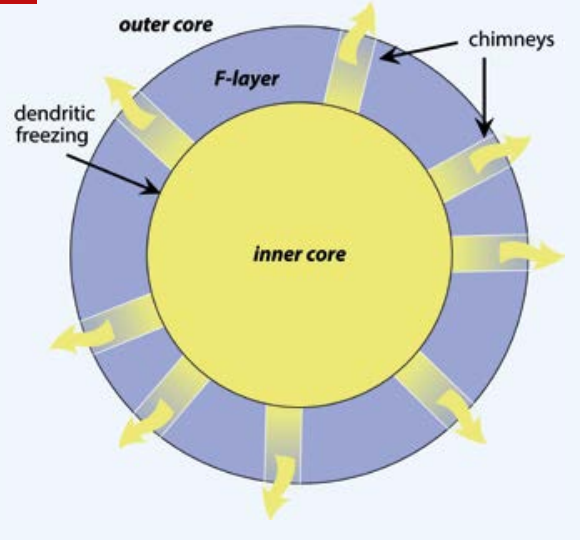

\section{Other planets}

More generally, stratified layers appear to be prevalent within planetary cores. While seismic observations of these layers exist only for the Earth, there are suggestions that several planets in our solar system may contain stably stratified layers in their electrically conducting regions - indeed this may be the case for all solar system bodies with active dynamos. A stably stratified layer underneath the convecting shell has been invoked for Uranus and Neptune in order to explain anomalous heat flow measurements, which suggest that large amounts of heat generated in their interiors must be trapped there (Hubbard et al. 1995). Also the structure of the magnetic fields of these planets is very different to that of other planets, including Earth; they are strongly non-dipolar and not axisymmetric. Numerical dynamo models of a convecting shell surrounding a stably stratified interior have been shown to create magnetic fields compatible with these observations (Stanley \& Bloxham 2006). Outer stably stratified layers surrounding the dynamo have also been inferred from recent Juno observations of Jupiter (Glatzmaier 2018) and proposed for Mercury and Saturn (Stanley \& Mohammadi 2008); they are necessary in order to reconcile $\mathrm{CMB}$ heat flow observations with evolutionary model predictions for Mercury, and to prevent Saturn from violating Cowling's theorem (Cowling 1934) despite its external field appearing axisymmetric. What causes these layers to form, and what their effects on core dynamics and dynamo action might be, is illuminated by what we know about the Earth and in turn can enlighten us about the behaviour of other planets.

\section{Summary}

It is clear that the deep Earth is a complex, abstruse place: the more closely we analyse it, the more intricacies we find. Layers of stratified fluid, although secondary to our classic picture of Earth's structure, do not necessarily play a subsidiary role in Earth's dynamics. While it is often tempting to isolate regions of the deep Earth, it is becoming increasingly clear that the interaction between both the inner and outer core, and the outer core and the mantle provide some of the most interesting and perhaps pivotal regions for the geodynamo, at both the base and the top of the outer core. These regions present active areas of research; properly understanding them, and how regions of the Earth are coupled together, will be key to a breakthrough in understanding Earth's magnetic field generation.

\section{AUTHORS}

Colin M Hardy is a PhD student at University of Leeds, UK.. Jenny Wong is a postdoctoral researcher at Institut de Physique du Globe de Paris (IPGP), France.

\section{ACKNOWLEDGEMENTS}

Colin M Hardy is supported by EPSRC grant no. EP/L01615X/1. Jenny Wong is supported by EP/ L01615X/1 and the Simone and Cino Del Duca Foundation of the Institut de France.

\section{REFERENCES}

Alboussière T et al. 2010 Nature 466744 Alexandrakis C \& Eaton DW 2010 Phys. Earth Planet. Inter. 180(1-2) 59

Alfè D et al. 2002a J. Chem. Phys. 1167127

Alfè D et al. 2002b Earth Planet. Sci. Lett. 19591 Badro J et al. 2014 Proc. Natl. Acad. Sci. 1117542 Bergman MI 1993 Geophys. Astrophys. Fluid Dyn. 68(1-4) 151

Braginsky SI 1963 Sov. Phys. Dokl. 1498

Braginsky SI 1984 Geophys. Astrophys. Fluid Dyn 30(1-2) 1

Braginsky SI 1987 Geomagn. Aeron. 27410

Braginsky SI 1993 J. Geomag. Geoelect. 45(11-12) 1517

Braginsky SI \& Roberts PH 1995 Geophys.

Astrophys. Fluid Dyn. 791

Brodholt J \& Badro J 2017 Geophys. Res. Letts

44(16) 8303

Buffett B A 2014 Nature 507(7493) 484

Buffett B A \& Seagle CT 2010 J. Geophys. Res. Solid

\section{Earth 115 B04407}

Bullen KE 1953 An Introduction to the Theory of Seismology (Cambridge University Press)

Christensen UR 2006 Nature 444(7122) 1056

Christensen UR \& Wicht J 2008 /carus 196(1) 16 Cleary JR \& Haddon RAW 1972 Nature 240(5383) 549

Cowling TG 1934 Mon. Not. R. Astron. Soc. 9439

Cox GA et al. 2018 arXiv:1807.00310

Davies CJ et al. 2015 Nature Geosci. 8(9) 678 Davies CJ et al. Earth Planet. Sci. Lett. in press de Koker N et al. 2012 Proc. Natl Acad. Sci. 109407 Deguen R et al. 2007 Phys. Earth Planet. Int. 16436 Deguen R et al.2018 Geophys. J. Int. 214(1) 88 Deuss A 2014 Ann. Rev. Earth Planet. Sci. 42103 Dziewonski AM \& Anderson DL 1981 Phys. Earth Planet. Int. 25297

Fearn DR et al. 1981 Nature 291232 Finlay CC et al. 2016 Earth, Planets and Space 68(1) 112

Glatzmaier GA 2018 Proc. Nat. Acad. Sci. 115(27) 6896

Gomi H et al. 2013 Phys. Earth Planet. Int. 22488 Gubbins D \& Davies CJ 2013 Phys. Earth Planet. Int. 21521

Gubbins D et al. 2003 Geophys. J. Int. 155609 Gubbins D et al. 2008 Geophys. J. Int. 1741007 Gubbins D et al. 2011 Nature 473361

Gubbins D 2007 Earth Planets Space 59(7) 661

Gutenberg B 1913 Phys. Z. 141217

Helffrich G 2012 Geophys. J. Int. 1881065

Helffrich G \& Kaneshima S 2010 Nature 468(7325) 807
Helffrich G \& Kaneshima S 2013 Phys. Earth

Planet. Int. 2232

Hirose Ket al. 2013 Ann. Rev. Earth Planet. Sci. 41(1) 657

Hubbard WB et al. 1995 in Neptune and Triton ed. DP Cruikshank (University of Arizona Press) 109 Huguet Let al. 2018 Earth Planet. Sci. Lett. 4879 Irving JCE et al. 2018 Science Advances 4(6) eaar2538

Jeffreys H 1939 Geophys. J. Int. 4594 Jones CA 2015 in Treatise on Geophysics (Elsevier) 8115

Kaneshima S 2018 Phys. Earth Planet. Int. 276234 Kennett BLN et al. 1995 Geophys. J. Int. 122108 Kind R \& Li X 2015 in Treatise on Geophysics (Elsevier) 1655

King DW et al. 1973 Earth Planet. Sci. Lett. 20(3) 353

Koper KD \& Pyle ML 2004 J. Geophys. Res. 109 B03301

Landeau M et al. 2016 Nature Geosci. 9(10) 786 Lay T \& Young CJ 1990 Geophys. Res. Lett. 17(11) 2001

Lehmann I 1936 Publ. Bur. Cent. Seismol. Int. 143 Lesur V et al. 2015 Geophys. J. Int. 201(2) 929 Loper DE \& Roberts PH 1977 Geophys. Astrophys. Fluid Dyn. 9289

Masters G \& Gubbins D 2003 Phys. Earth Planet. Int. 140159

Moffatt HK \& Loper DE 1994 Geophys. J. Int. 117394

Mound J et al. 2017 The apparent stratification at the top of Earth's liquid core EarthArXiv doi:10.31223/osf.io/dvfjp

Mullins WW \& Sekerka RF 1964 J. App. Phys. 35444

Nakagawa T 2018 Phys. Earth Planet. Int. 276172 Nakajima Y et al. 2015 Nature Comms 68942 Nimmo F 2007 in Treatise on Geophysics (Elsevier) 9217

Oldham RD 1906 Quart. J. Geol. Soc. 62(1-4) 456 Olson Pet al. 2018 Frontiers Earth Sci. 6140 Pozzo M et al. 2012 Nature 485355 Roberts PH \& Glatzmaier G A 2000 Rev. Mod. Phys. 72(4) 1081

Roberts PH et al. 2007 Geophys. Astrophys. Fluid Dyn. 101(1) 11

Song X \& Richards PG 2006 Nature 382221 Souriau A 2007 in Treatise on Geophysics (Elsevier) 1655

Souriau A \& Poupinet G 1991a Geophys. Res. Lett. 182023

Souriau A \& Poupinet G 1991b Phys. Earth Planet. Int. 68(1-2) 183

Stanley S \& Bloxham J 2006 / carus 184556 Stanley S \& Mohammadi A 2008 Phys. Earth Planet. Int. 168(3-4) 179

Tackley PJ 2007 in Treatise on Geophysics (Elsevier) 7437

Tkalčić H et al. 2009 Geophys. J. Int. 179425 Umemoto K \& Hirose K 2015 Geophys. Res. Lett. 42(18) 7513

Vallis GK 2017 Atmospheric and Oceanic Fluid Dynamics (Cambridge University Press)

Wong J et al. 2018 Geophys. J. Int. 214(3) 2236

Zou Z et al. 2008 J. Geophys. Res. 113 B05314 